EGU21-7730, updated on 16 Nov 2021

https://doi.org/10.5194/egusphere-egu21-7730

EGU General Assembly 2021

(c) Author(s) 2021. This work is distributed under

the Creative Commons Attribution 4.0 License.

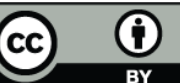

\title{
European Underground Laboratories Association EUL - An International Partner for Underground Research Opportunities
}

\author{
Helmut Mischo ${ }^{1}$, Krzysztof Fuławka ${ }^{2}$, and Jari Joutsenvaara ${ }^{3}$ \\ ${ }^{1}$ Technical University Bergakademie Freiberg, Mining Engineering, Underground Mining Methods, Freiberg, Germany \\ (helmut.mischo@mabb.tu-freiberg.de) \\ ${ }^{2}$ KGHM Cuprum, Poland \\ ${ }^{3}$ University of Oulu. Finland
}

Underground experimental sites and laboratories are rare and offer unique opportunities for research, development, innovation, education and training, among other usages due to their special boundary conditions. Each underground facility is highly unique in its geological and geophysical characteristics. These sites can be either dedicated infrastructures built for specific usage or mines and parts of mines freed from underground extraction. Since they are usually isolated from environmental influences and, conversely, shield experiments as far as possible from the environment, they offer unique research conditions and possibilities compared to surface laboratories.

However, the sustainable operation of such underground experimental sites is not an easy task. Hence, to foster and accommodate the use, scientific collaboration and interdisciplinary scientific research among European underground research facilities, a specialized association has been set up by a number of European partners under the name European Underground Laboratories Association EUL.

This association defines its purpose by forming a network between the underground laboratories and client organizations from business, science and administration sectors. The goal is to bundle and develop existing competencies, and thus providing a common platform for members and prospective researchers and costumers on order to share and exchange information and experience, and in turn to contribute to the development and implementation of new research projects. As an Europe-wide and internationally active association comprising EU members and non-members, EUL also supports and promotes European integration and international cooperation.

This paper provides an overview over the structure and the organisation of EUL, its member institutions and associated underground research laboratories as well as the possibilities the association may offer for its members and interested partners in the fields of:

- providing a comprehensive overview of the research possibilities and conditions at the different underground sites to clients and the public 
- infrastructure development at the research locations and improvement of research conditions in each underground facility

- execution of joint research proposals and respective project planning

- setting up and extending the spread of advertising materials and publications

- education and training of students, researchers and professionals,

- enabling the exchange of researchers, professionals and/or students among partnering facilities 